\title{
Tumeur de Pott compliquant une sinusite frontale
}

\section{Pott's Puffy Tumor, Complication of a Frontal Sinusitis}

\section{G. Penhouët $\cdot$ O. Betoux $\cdot$ O. Croizier}

Reçu le 30 mars 2015; accepté le 15 mai 2015

(C) SFMU et Lavoisier SAS 2015

Un patient de 45 ans s'est présenté aux urgences pour des céphalées. L'examen aux urgences retrouvait une masse frontale gauche rénitente indolore, un œdème inflammatoire péri-orbitaire gauche (Fig. 1A), une douleur de l'épicanthus interne de l'œil droit et une rhinorrhée purulente postérieure. Le bilan biologique objectivait le syndrome infectieux : hyperleucocytose (GB 15,99G/L), CRP à $33 \mathrm{mg} / \mathrm{L}$. Une ponction à visée exploratrice de la masse frontale a confirmé la nature infectieuse de la lésion et a permis l'évacuation de $14 \mathrm{ml}$ d'un liquide purulent. L'analyse bactériologique a mis en évidence Fusobacterium SPP, bacille Gram négatif anaérobie, germe saprophyte de l'oropharynx. La tomodensitométrie retrouvait une sinusite maxillo-ethmoïdo-frontale à prédominance gauche avec ostéolyse des tables externe et interne des régions frontales responsables d'abcès sous périostés endocrâniens bifrontaux, soit une ostéomyélite frontale ou tumeur de Pott (Fig. 1B). Le traitement a consisté en un drainage chirurgical par clou de Lemoyne de la sinusite frontale et une antibiothérapie pendant sept semaines. Le traitement a permis une évolution favorable. La tumeur de Pott est une complication rare de la sinusite frontale depuis l'apparition des antibiotiques et nécessite un traitement chirurgical avec drainage de l'abcès. Cette pathologie concerne majoritairement les enfants et les adultes jeunes, et prédomine chez les hommes [1,2]. Les analyses bactériologiques identifient un streptocoque ou un staphylococcus aureus dans près d'un cas sur deux. Les complications intracrâniennes surviennent le plus souvent chez l'enfant.

Liens d'intérêts : Les auteurs déclarent ne pas avoir de lien d'intérêt.

G. Penhouët $(\bowtie) \cdot$ O. Betoux $\cdot$ O. Croizier

Service d'accueil des urgences-SMUR, centre hospitalier de Cholet, 1 rue Marengo, F-49325 Cholet cedex, France e-mail : gaellepenhouet@hotmail.com
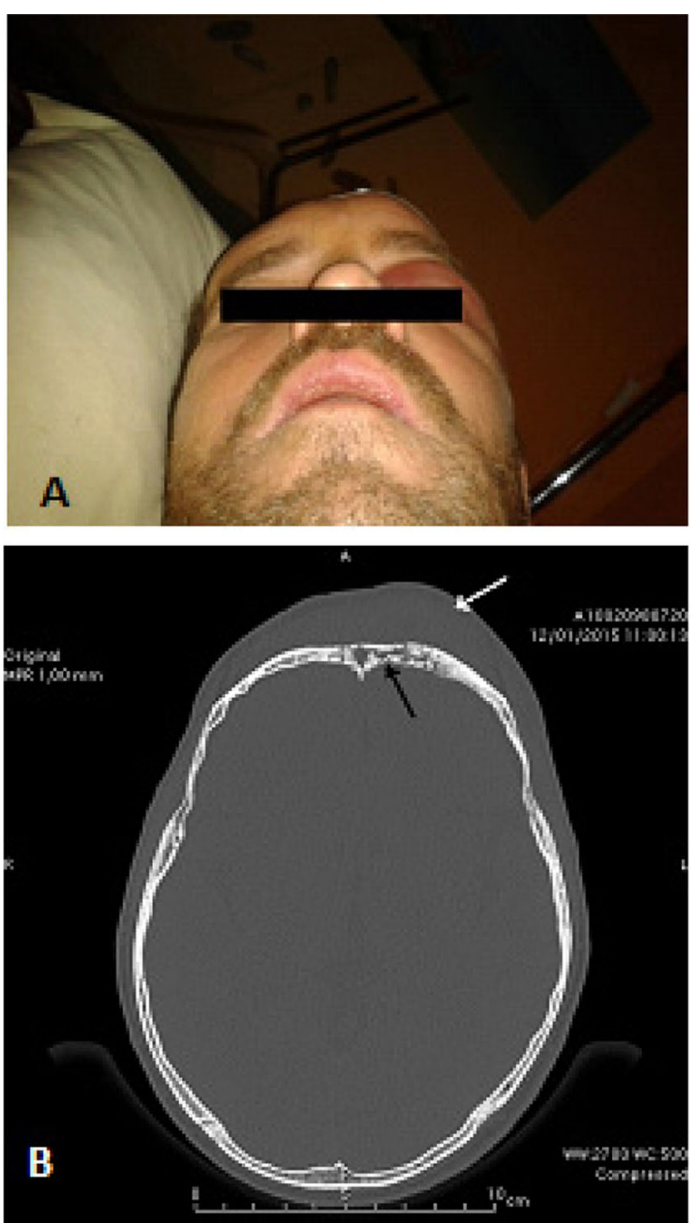

Fig. 1 A : abcès sous-cutané frontal gauche et œdème palpébral gauche chez un patient de 45 ans ; B : scanner cérébral sans injection en coupe axiale, montrant une ostéomyélite de l'os frontal gauche (flèche noire) et abcès sous-cutané (flèche blanche)

\section{Références}

1. Skomro R, McClean KL (1998) Frontal osteomyelitis (Pott's Puffy Tumor) associated with pasteurella multocida - A case report and review of the literature. Can J Infect Dis 9:115-21

2. Jung J, Lee HC, Park IH, Lee HM (2012) Endoscopic endonasal treatment of a Pott's Puffy Tumor. Clin Exp Otorhinolaryngol 5:112-5 